\title{
Power spectrum of the current in systems with a conserved density
}

\author{
Kwan-tai Leung* \\ Department of Physics, Virginia Polytechnic Institute and State University, Blacksburg, Virginia 24061
}

(Received 1 April 1991; revised manuscript received 28 May 1991)

\begin{abstract}
Motivated by recent studies of model sandpiles, the power spectrum $S$ of the current in dissipative dynamical systems with a conserved density is investigated. In contrast to self-organized criticality exhibited in certain lattice gases and noisy Langevin equations, where power laws are described by universal, simple indices, the index of $S(f)$ for small frequency $f$ is determined at a second-order phase transition by nontrivial critical exponents. For systems with no external drive (i.e., model $B$ ), the exact result for the dynamic exponent $z$ is rederived. With drive (driven diffusive systems), the index is given by the exponent of anisotropies. Simulation in two dimensions yields good agreements with theoretical predictions.
\end{abstract}

Recently, there has been considerable interest in the studies of power spectra for dynamical systems exhibiting temporal and spatial scale invariance. Such an interest is inspired by the idea of self-organized criticality ${ }^{1}$ which is an attempt to provide an explanation for the ubiquity of power-law behavior, particularly the occurrence of $1 / f$ noise, ${ }^{2}$ found in dissipative dynamical systems. Within that framework, it is generally argued that a dissipative system driven out of equilibrium exhibits generic powerlaw behavior without any parameter tuning.

Apart from sandpile models, ${ }^{1,3}$ several nonequilibrium lattice-gas models were also found to show some of the same characteristics. ${ }^{4}$ Since the total number of particles, $N$, is not conserved in those models, its temporal fluctuation is of obvious physical interests. The power spectra of $N$ have been shown to display $1 / f$ behavior, insensitive to a wide range of physical parameters and independent of the spatial dimensions. The driven diffusive systems represent another important class of nonequilibrium lattice gas. $^{5}$ In addition to nearest-neighbor interaction among the particles, the system is driven out of equilibrium by an external driving force which acts locally like a uniform electric field. Dissipation is introduced by coupling the system to a heat bath which absorbs the energy input by the field. Far above the critical temperature $T_{c}$, powerlaw behavior prevails by virtue of the physical argument offered by self-organized criticality. ${ }^{6}$ However at $T_{c}$ power laws are modified by critical fluctuations. In general, power laws are then characterized by nontrivial critical exponents. This is in contrast to certain lattice gases and noisy, Lagevin equations which yield integral or halfintegral indices ${ }^{4,6}$ like $1, \frac{3}{2}$, or 2 .

The driven diffusive systems belong to the class of systems having locally conserving bulk density. For lattice gases, this refers to the case in which the local dynamics strictly conserves the number of particles. For Langevin theories, this refers to the case in which the bulk density strictly obeys a continuity equation, hence with vanishing noise correlation at zero momenta. ${ }^{7}$ For constant total number of particles, the simplest power spectrum $S(\omega)$ to consider is that of the current density $J$. In this paper, the scaling behavior of this quantity at $T_{c}$ for the driven diffusive systems with attractive interaction ${ }^{5}$ is derived by scaling arguments, and numerical results from Monte Carlo simulation in two dimensions are presented. As expected, the exponent $\varphi$ defined in $S(\omega) \sim \omega^{-\varphi}$ for small frequency $\omega$ is completely determined by critical exponents, in particular the dynamic exponent ${ }^{8} z$. $S$ has also been computed in low spatial dimensions $(d \leq 2)$ in the high-temperature, homogeneous phase, where anomalous, nondiffusive behavior was found. ${ }^{9}$

In the presence of an external drive, the current along the drive is finite. Its spatial average normal to a unit area is a temporally fluctuating quantity given by $Q(t)$ $\equiv V^{-1} \sum_{x \|} \sum_{x \perp} J_{\|}(x, t)=V^{-1} \tilde{J}_{\|}\left(k_{\|}=0, k_{\perp}=0, t\right)$, i.e., the spatial Fourier transform of $J_{\|}$, with $V$ the volume. Here the coordinates are labeled with respect to the direction of the drive. The power spectrum

$$
\begin{aligned}
S(\omega) & =\lim _{\tau \rightarrow \infty} \frac{1}{\tau}\left\langle\left|\sum_{t=1}^{\tau} Q(t) e^{i \omega t}\right|^{2}\right\rangle \\
& =V^{-1} \frac{\left\langle\tilde{J}_{\|}(k=0, \omega) \tilde{J}_{\|}\left(k^{\prime}=0, \omega^{\prime}\right)\right\rangle}{\delta\left(k+k^{\prime}\right) \delta\left(\omega+\omega^{\prime}\right)} \\
& =V^{-1} G_{J J}(k=0, \omega)
\end{aligned}
$$

is defined as an ensemble average in the steady state.

In a continuum description, the coarse-grained particle density $\phi(x, t)$ obeys a continuity equation

$$
\frac{\partial \phi}{\partial t}=-\nabla \cdot \mathbf{J}
$$

Thus, $S(\omega)$ can be obtained from the density correlation function through

$$
S(\omega)=V^{-1} \omega^{2} \lim _{k_{\|} \rightarrow 0} k_{\|}^{-2} G_{\phi \phi}\left(k_{\|}, k_{\perp}=0, \omega\right) .
$$

The $J-J$ correlations along directions other than the longitudinal one vanish upon taking $k_{\perp} \rightarrow 0$. The scaling behavior of $S(\omega)$ can then be derived from the known properties of $G_{\phi \phi}$ at $T_{c}{ }^{10,11}$

For simplicity, let us first consider the equilibrium systems with no drive, i.e., model $B .^{8}$ Replacing $k_{\|}$in Eq. (3) by $k$ in the absence of anisotropy, we have for small $\omega$

$$
\begin{aligned}
S(\omega) & =V^{-1} \omega^{2} \lim _{k \rightarrow 0} k^{-2} k^{-2+\eta-z} \bar{G}\left(k^{-z} \omega\right) \\
& \sim V^{-1} \omega^{(z-4+\eta) / z} .
\end{aligned}
$$


Here $\eta$ and $z$ are the usual correlation-function and dynamic exponents. The last line follows from the singularity of the scaling function $\bar{G}(x) \sim x^{(-4+\eta-z) / z}$ as $x \rightarrow \infty$, because $S(\omega)$ is positive definite and finite. With no external drive, $\langle J\rangle=0$, and $Q(t)$ at different times are uncorrelated. To see these, recall that the current density for model $B$ is ${ }^{8}$

$$
\mathbf{J}=-\Gamma \nabla \frac{\delta \mathcal{H}}{\delta \phi}+\mathbf{j},
$$

with the first (second) term being the deterministic (random) part, $\Gamma$ the transport coefficient, and $\mathscr{H}$ the usual Landau-Ginzburg-Wilson Hamiltonian. Thus, only the random noise $\mathrm{j}$ contributes to $\tilde{J}(k \rightarrow 0, t)$. Since $\mathrm{j}$ has Gaussian distribution with zero mean,

$$
\left\langle j_{\alpha}(x, t) j_{\beta}\left(x^{\prime}, t^{\prime}\right)\right\rangle=2 \Gamma T \delta_{\alpha \beta} \delta\left(x-x^{\prime}\right) \delta\left(t-t^{\prime}\right),
$$

a white-noise spectrum ( $S$ independent of $\omega$ ) for $Q$ follows for all temperature. This is easily verified numerically for the Ising lattice gas (see Fig. 1). Moreover, the linear $T$ dependence in Eq. (6) is found to be valid over a wide range of $T$. Consequently, for $S(\omega)$ in Eq. (4) to be independent of $\omega$, the exact result ${ }^{8} z=4-\eta$ is rederived.

Returning to the driven systems, the scaling form of the correlation function at $T_{c}$ was obtained by treating critical fluctuations in a dynamic renormalization-group analysis. ${ }^{10}$ It is governed by strong anisotropies:

$$
G_{\phi \phi}\left(k_{\|}, k_{\perp}, \omega\right)=k_{\perp}^{-2+\eta_{\perp}-z_{\perp}} \bar{G}\left(k_{\|} k_{\perp}^{-\lambda}, k_{\perp}^{-z_{\perp} \omega}\right),
$$

in which $\lambda \neq 1$ is the major source of subtlety of the associated finite-size effects. ${ }^{11,12}$ Using this, a finite right-hand side of Eq. (3) in the limit $k_{\|} \rightarrow 0$ demands $S$ to scale in the low frequency limit as

$$
S(\omega) \sim V^{-1} \omega^{-\varphi},
$$

where $\varphi=\left(2-\eta_{\perp}+2 \lambda-z_{\perp}\right) / z_{\perp}$. This result is generally valid for systems satisfying Eq. (2), i.e., with strictly conserving density. Clearly, $\varphi$ is specific to the relevant terms in $J(\phi)$ in Eq. (2). It has been determined theoretically for the driven diffusive systems that the transverse ex- ponents are mean-field like: ${ }^{10,13} z_{\perp}=4$ and $\eta_{\perp}=0$, for all spatial dimensions $d$ between 2 and 5. The simple result $\varphi=(\lambda-1) / 2$ thus follows. Therefore, this is a direct way to measure the important exponent for spatial anisotropy, $\lambda$, which plays a crucial role in the finite-size scaling near criticality. Using again the field-theoretic result ${ }^{11} \lambda=(11$ $-d) / 3, \varphi$ is found to interpolate between 1 and $\frac{1}{2}$ as $d$ changes from 2 to 5 .

Simulation results. Monte Carlo simulations have been carried out to test this prediction. In $d=2$, the lattice gas is defined on a square lattice of sizes $L \times L$, updated according to the Metropolis jump rates with strongly biased hopping (spin exchange) dynamics. Since the simulations have been described in detail elsewhere, ${ }^{5}$ there is no need to repeat it here. With temperature fixed at $T_{c}$ ( $\approx 1.42$ in units of the Onsager critical temperature, as determined recently by finite-size scaling $\left.{ }^{11}\right)$, the power spectrum of the averaged current $Q(t)$ is computed in the steady state with a length of time series $\tau$ ranging from $2^{10}$ to $2^{16}$ sweeps, averaged over from 50 to 100 runs, for $L=30,50$, and 80 . Taken together, an assessment of the effects of finite time sequences and finite system sizes can thus be made.

Figures 2 and 3 are $\log _{10}-\log _{10}$ plots of $S(\omega)$ against $f=\omega / 2 \pi$, showing evidence of power law at relatively low frequency (in comparison to other models ${ }^{4}$ ). Since, for finite systems, this asymptotic behavior does not extend over many decades, a log-log plot may be misleading. But by plotting $1 / S$ vs $f$, a linear behavior at small $f$ is clearly shown. This justifies the use of log-log plot.

Near $T_{c}$, a slight dependence of $\varphi$ on temperature is detected. As $T$ approaches $T_{c} \approx 1.42$ from either side, $\varphi$ converges to unity, in agreement with Eq. (8), as exemplified by Fig. 2. For $T$ well below $T_{c}$, a power-law description seems to break down with the appearance of a "shoulder" at some intermediate frequency, signaling a time scale is in play. Such a time scale apparently has to do with the presence of domain walls. $\varphi$ continues to decrease as $T$ is increased beyond $T_{c}$, presumably reaching the asymptotic logarithmic behavior in the infinite- $T$ limit. ${ }^{9}$ Unfortunately, the data in this limit are too noisy to

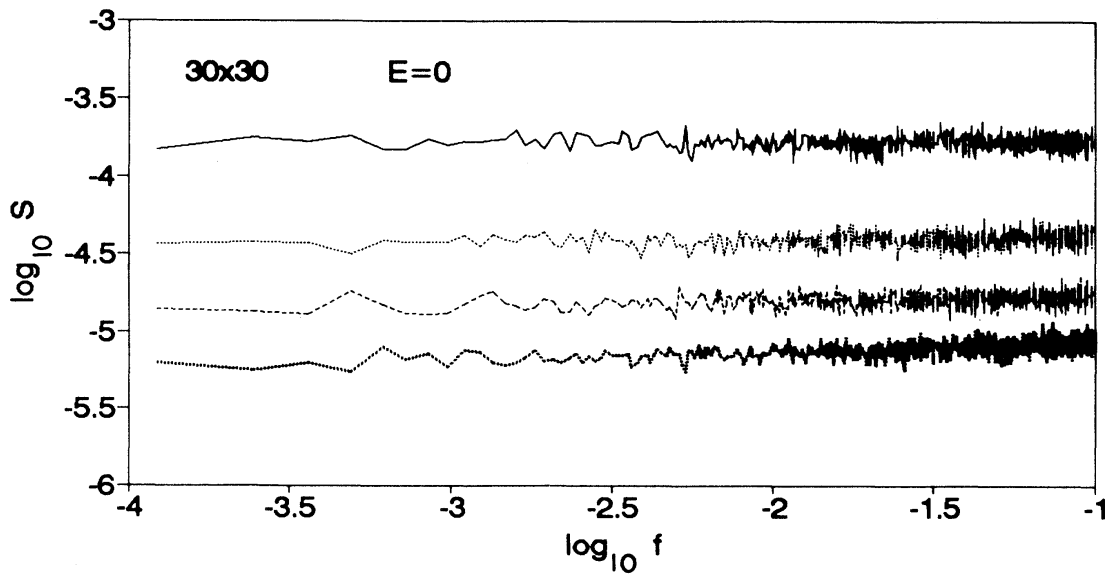

FIG. 1. A $\log _{10}-\log _{10}$ plot of the power spectrum $S(f)$ of the averaged current vs frequency $f$ for the equilibrium Ising lattice gas (with $E=0$ ) in a wide range of temperature: $T=3,1.05,0.8$, and 0.65 , from top to bottom, in units of $T_{c}(E=0)$. 


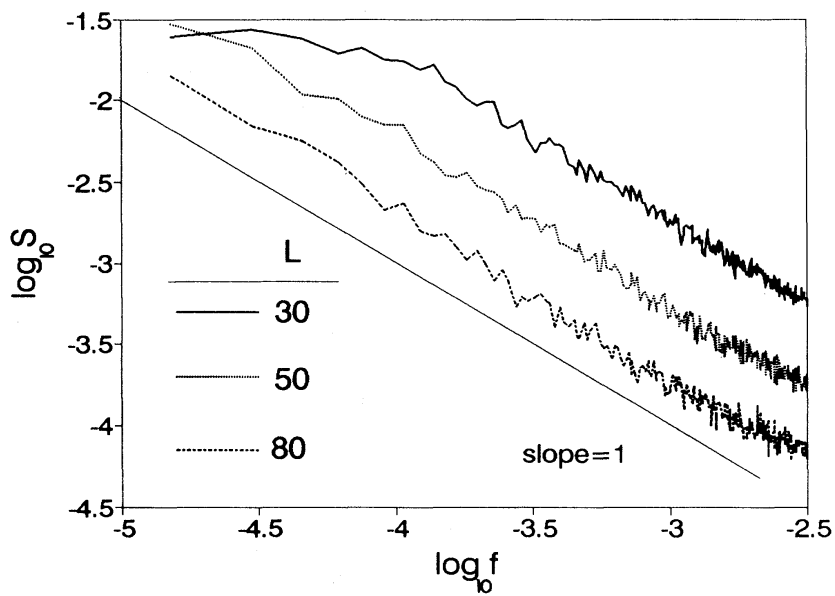

FIG. 2. $S(f)$ for the averaged current vs frequency $f$ for the driven lattice gas at $T=T_{c}$, where $T_{c} \approx 1.42$. The straight line serves as a comparison. The vertical displacement of the curves for different system sizes is due to the $\log _{10} V$ factor in Eq. (6). Finite-size effects are only noticeable at low frequencies.

resolve the difference between a logarithmic behavior and a power law with a very small exponent.

By finite-size scaling, ${ }^{11,12} L_{\|}$and $L_{\perp}$ are also related by the exponent $\lambda$ via a scaling variable $L_{\|} L_{\perp}^{-\lambda}$. $\lambda$ in this form has also been estimated ${ }^{14}$ to be about 2.24 , which corresponds to $\varphi \approx 0.62$. This disagrees decisively with the above data and Eq. (8). The origin of such a discrepancy clearly lies in the previous uncertainty in $T_{c}$.

To conclude, analyzing the power spectrum of the current in driven diffusive models not only demonstrates quantitatively the difference between thermodynamic critical scaling and self-organized criticality (within the context of models as studied in Refs. 4 and 6), but also yields

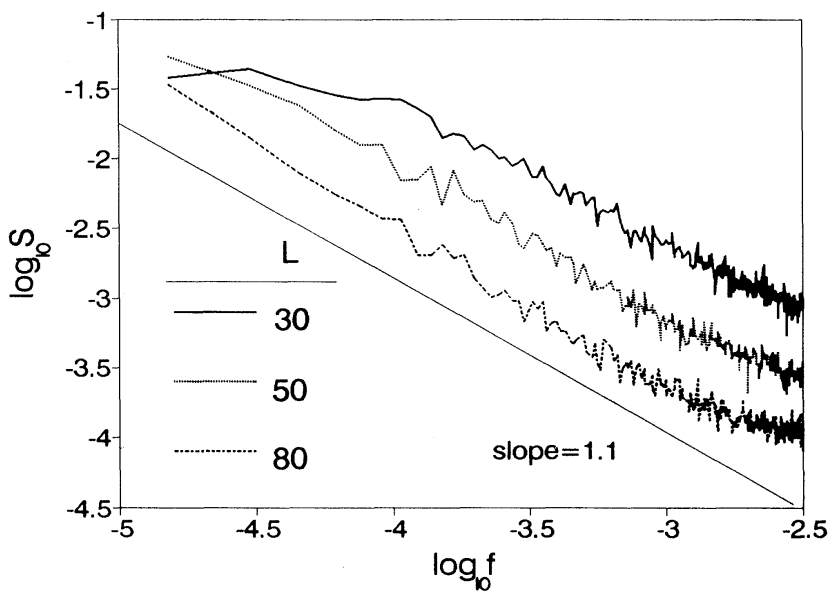

FIG. 3. Same as Fig. 2, but for $T=1.38$, slightly below $T_{c}$. The slopes are slightly greater than one, whereas they are somewhat smaller than one for $T$ slightly above $T_{c}$ (not shown).

valuable information on critical exponents, relatively free from subtle finite-size effects. For systems with no drive, this analysis simply reproduces the exact result for the dynamic exponent $z$. Studying power spectra for other physical quantities should also prove useful in obtaining reliable estimates of critical exponents.

This work is supported by the National Science Foundation under Grant No. DMR-8817653. This research was conducted using the Cornell National Supercomputer Facility, a resource of the Cornell Theory Center, which is funded in part by the National Science Foundation, New York State, the IBM Corporation, and members of the Center's Corporate Reserach Institute.

${ }^{*} E$-mail: rkpzia@vtvm1.

1(a) P. Bak, C. Tang, and K. Wiesenfeld, Phys. Rev. Lett. 59, 381 (1987); Phys. Rev. A 38, 364 (1989); (b) T. Hwa and M. Kardar, Phys. Rev. Lett. 62, 1813 (1989); G. Grinstein, D.-H. Lee, and S. Sachdev, ibid. 64, 1927 (1990).

${ }^{2}$ See, e.g., W. H. Press, Commun. Mod. Phys. C 7, 103 (1978); M. B. Weissman, Rev. Mod. Phys. 60, 537 (1988), and references therein.

${ }^{3}$ H. J. Jensen, K. Christensen, and H. C. Fogedby, Phys. Rev. B 40, 7425 (1989)

${ }^{4}$ H. J. Jensen, Phys. Rev. Lett. 64, 3103 (1990); J. V. Andersen, H. J. Jensen, and O. G. Mouritsen (unpublished); G. Grinstein, T. Hwa, and H. J. Jensen (unpublished).

${ }^{5}$ S. Katz, J. L. Lebowitz, and H. Spohn, Phys. Rev. B 28, 1655 (1983); J. Stat. Phys. 34, 497 (1984); J. L. Vallés and J. Marro, J. Stat. Phys. 43, 441 (1986); 49, 89 (1987).

${ }^{6}$ M. Q. Zhang, J.-S. Wang, J. L. Lebowitz, and J. L. Vallés, J.

Stat. Phys. 52, 1461 (1988); P. L. Garrido, J. L. Lebowitz, C. Maes, and H. Spohn, Phys. Rev. A 42, 1954 (1990).

${ }^{7}$ See Ref. 1(b) for Langevin models in the context of selforganized criticality, with and without conservative noise.

${ }^{8}$ P. C. Hohenberg and B. I. Halperin, Rev. Mod. Phys. 49, 435 (1977).

${ }^{9}$ H. van Beijeren, R. Kutner, and H. Spohn, Phys. Rev. Lett. 54, 2026 (1985); H. K. Janssen and B. Schmittmann, Z. Phys. B 63, 517 (1986).

${ }^{10}$ K.-t. Leung and J. L. Cardy, J. Stat. Phys. 44, 567 (1986); H. K. Janssen and B. Schmittmann, Z. Phys. B 64, 503 (1986).

${ }^{11}$ K.-t. Leung, Phys. Rev. Lett. 66, 453 (1991).

${ }^{12}$ K. Binder and J.-S. Wang, J. Stat. Phys. 55, 87 (1989).

${ }^{13}$ H. van Beijeren and L. S. Schulman, Phys. Rev. Lett. 53, 806 (1984).

${ }^{14}$ J.-S. Wang, K. Binder, and J. L. Lebowitz, J. Stat. Phys. 56, 783 (1989). 